\title{
Brain Connectivity in Positive and Negative Syndrome Schizophrenia
}

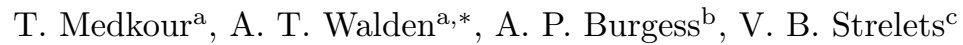 \\ ${ }^{a}$ Department of Mathematics, Imperial College London, 180 Queen's Gate, London SW7 \\ 2BZ, UK \\ ${ }^{b}$ Aston University School of Life and Health Sciences, Aston Triangle, Birmingham B4 \\ ZET, UK \\ ${ }^{c}$ Institute of Higher Nervous Activity and Neurophysiology, Russian Academy of Sciences, \\ Butlerov Street 5 A, 117485 Moscow, Russia
}

\begin{abstract}
If, as is widely believed, schizophrenia is characterised by abnormalities of brain functional connectivity, then it seems reasonable to expect that different subtypes of schizophrenia could be discriminated in the same way. However, evidence for differences in functional connectivity between the subtypes of schizophrenia is largely lacking and, where it exists, it could be accounted for by clinical differences between the patients (e.g. medication) or by the limitations of the measures used. In this study, we measured EEG functional connectivity in unmedicated male patients diagnosed with either positive or negative syndrome schizophrenia and compared them with age and sex matched healthy controls. Using new methodology (Medkour et al., 2009) based on partial coherence, brain connectivity plots were constructed for positive and negative syndrome patients and controls. Reliable differences in the pattern of functional connectivity were found with both syndromes showing not only an absence of some of the connections that were seen in controls but also the presence of connections that the controls did not show. Comparing connectivity graphs using the Hamming distance, the negative-syndrome patients were found to be more distant from the controls than were the positive syndrome patients. Bootstrap distributions
\end{abstract}

\footnotetext{
*Corresponding author. $\quad$ Tel: $\quad$ (0)20 7594 8524; Fax: (0)20 $7594 \quad 8517$; e-mail: a.walden@imperial.ac.uk
} 
of these distances were created which showed a significant difference in the mean distances that was consistent with the observation that negative-syndrome diagnosis is associated with a more severe form of schizophrenia. We conclude that schizophrenia is characterised by widespread changes in functional connectivity with negative syndrome patients showing a more extreme pattern of abnormality than positive syndrome patients.

Key words: Brain connectivity, Graphical models, Partial spectral coherence, Schizophrenia

\section{Introduction}

Bleuler's original conception of schizophrenia as a disease caused by disconnections between the fundamental components of the mind has, after a long hibernation, undergone a process of revival and modernisation and been reframed in terms of an abnormality of functional connectivity in the brain (Friston and Frith, 1995). So successful has this revival been that the disconnection hypothesis has become one of the most active areas of schizophrenia research (Bullmore and Fletcher, 2003). In part the renaissance of the disconnection hypothesis can be viewed as a natural consequence of technological developments that have made possible the measurement of both structural (Diffusion Tensor Imaging) and functional connectivity in vivo. Perhaps more important, however, has been the growing understanding of the role of synchronous cerebral oscillations as a neural code that enables visual feature binding and, by extension, permits functional integration across the brain more generally. From here, it is but a small step to propose that schizophrenia should be characterised by abnormalities in the pattern of synchronous cerebral oscillations and numerous studies have been reported that set out to test this hypothesis.

There is certainly consistent evidence of abnormal cerebral oscillations in schizophrenia. Patients typically show unusually high EEG power in the low frequencies (delta and theta bands, $<8 \mathrm{~Hz}$ ) which is usually interpreted as evidence of neurodevelopmental delay (Galderisi et al., 2009), but this difference is 
by no means specific to schizophrenia (Coutin-Churchman et al., 2003). Furthermore, although EEG power at single scalp sites reflects localised synchronous neuronal activity, it does not provide a useful index of more long-distance functional connectivity and to do this, some measure of inter-channel covariation is required, such as coherence or phase synchrony. Much of the early work on functional connectivity in schizophrenia focussed on EEG coherence in the classical frequency bands (delta, theta, alpha and beta) and although abnormalities were claimed, the direction and locus of the changes reported were inconsistent (Leocani and Comi, 1999). More recent studies, perhaps influenced by proposed role of gamma oscillations in feature binding, have examined high frequency connectivity using a plethora of measures of connectivity and but have come to broadly similar conclusions (Stephan et al., 2006; Uhlhaas et al., 2008): although there is consistent evidence for the aberrance of functional connectivity mediated through cortical oscillations in schizophrenia, the nature and direction of the abnormality is uncertain.

This confusing state of affairs may reflect the true heterogeneity of the disorder and the samples of patients examined. It might, however, reflect differences between patients that arise secondary to the diagnosis of schizophrenia, such as medication, rather than to the diversity of the disease itself. EEG measures of functional connectivity also face fundamental methodological challenges that, although well known, have not been adequately addressed. These include the problems of volume conduction and that of multiple comparisons.

The problem of volume conduction is that if there is a significant level of connectivity between two channels, is it due to a flow of information between two or more spatially separate regions or because the sensors are detecting activity emanating from a single source? Most measures of connectivity, including coherence, phase synchrony and all current non-linear measures are unable to discriminate between these alternatives which means that they are not true measures of connectivity at all. Some measures, such as imaginary coherence (Nolte et al., 2004) or phase lag index (Stam et al., 2007) do successfully mitigate the volume conduction issue but invariably underestimate the 'true' connectivity. If 
it was possible to solve the inverse problem, then the volume conduction problem would become tractable but, unfortunately, source localisation can only be 'solved' by making strong assumptions about the nature of the sources to be localised and these assumptions invariably affect the estimates of connectivity between sources.

Medkour et al. (2009) developed a method for determining functional connectivity from partial coherence. Among the attractive properties of partial coherence are that it makes no assumptions about the nature of cerebral sources and markedly reduces the impact of volume conduction. Given these strengths, it is not surprising that partial coherence has been used before in EEG research but in all previous variants, the estimates of connectivity were unreliable. The revised approach carefully considers issues such as spectral matrix inversion, side-lobe leakage suppression, bias removal and multiple hypothesis testing for dependent partial coherencies, all aimed at increasing the reliability of connectivity results.

The clinical problems of the heterogeneity of schizophrenia and the impact of pharmaceutical treatments on functional connectivity also make interpretation of the studies problematic. The issue of medication is that any differences seen between patients and controls might well be due to the psychotropic effects of the medication rather than to the effects of the disease itself. The heterogeneity of schizophrenia is widely recognised clinically and, although there is no consensus, at least two syndromes are widely acknowledged: positive and negative (Andreasen and Olsen, 1982). Relatively little work has attempted to validate the positive/negative distinction using EEG but the finding that negative patients show greater delta and theta power than positive syndrome patients has been consistently reported (Begic et al., 2000, 2009; E. R. John et al., 1994; J. P. John et al., 2009). Furthermore, studies investigating Liddle's 3-cluster subtyping of schizophrenia has also found associations between delta power and Liddle's Psychomotor Poverty factor, which measures symptoms that are predominantly negative symptoms (Harris et al., 2001; Gross et al., 2006). Only two studies have examined functional connectivity measures in this context and 
both reported differences between positive and negative syndromes at high frequencies (Strelets et al., 2002; Lee et al., 2003) with negative patients showing lower levels of power.

The aim of this study was to determine whether it is possible to discriminate between positive and negative schizophrenia using the new methods of Medkour et al. (2009) - briefly discussed above, and referred to appropriately in what follows - for the determination of EEG functional connectivity that overcome many of the problems associated with previous approaches. New modelling and bootstrap resampling steps are developed in the current paper. Because previous research has found consistent differences at low frequencies, it was decided to focus on the delta frequency range, $[0.5,4] \mathrm{Hz}$, using a sample of unmedicated patients.

The paper is structured as follows. Firstly we discuss the subjects and EEG data. For clarity, we then divide up our methodology and results into three parts. Firstly, we give background details on the spectral matrix estimation method, and partial coherence calculation, its debiassing, and use in constructing a connectivity graph. Next we turn to defining the strength of connections through a measure we call 'total weighted relative strength,' and give a gamma distribution model for it. By using a threshold, this distribution is used to construct a connectivity graph, represented by a connection vector of ones and zeros. Finally, using the Hamming distance measure we show that, over a range of thresholds, the graph for negative-syndrome patients is further from the graph for controls than is the graph for positive-syndrome patients. Bootstrap resampling shows that this finding is statistically significant.

\section{Data}

\section{Subjects}

The data sample (see also Strelets et al. (2002)) was acquired from 34 male forensic patients (mean age 35; range 20-60) with a DSM-III-R diagnosis of schizophrenia from the Serbsky Institute in Moscow. Of these patients, 15 
showed a clear predominance of positive symptoms - delusions, hallucinations (Andreasen, 1984) and 19 had negative symptoms - personality defect, emotional retardation (Andreasen, 1983). (Patients with a mixed clinical picture were omitted from the study.) All patients were medication free or withdrawn from medication for at least 7 days before examination. The control group consisted of 24 healthy volunteers of comparable age to the patients (mean age $=$ 31; range 19-42). All subjects gave written informed consent for the investigation. Ethical approval came from the local Moscow ethics committee, and in compliance with national legislation and the Declaration of Helsinki. We shall denote the set of patients diagnosed as positive and negative syndromes by $\mathcal{P}$ and $\mathcal{N}$, respectively, and the controls by $\mathcal{C}$.

\section{EEG data and initial treatment}

EEG was recorded using an MBM (Russia) EEG mapper from 10 scalp sites (F3, F4, C3, C4, T3, T4, P3, P4, O1 and O2) referenced to linked ears with a bandpass filter of $0.5-45 \mathrm{~Hz}$. EEG recordings were obtained for resting conditions with eyes closed. A sampling rate of $100 \mathrm{~Hz}$, (sample interval $\Delta t=0.01 \mathrm{~s}$ ), was used so that the Nyquist frequency is $f_{\mathcal{N}}=1 /(2 \Delta t)=50 \mathrm{~Hz}$. While it would have been desirable to have more prefrontal and cortex sites, and a higher sampling rate (enabling examination of the gamma band), these were not available in this rare heritage clinical data set from unmedicated patients.

The alpha rhythm creates a characteristic and dominant spectral line at a frequency of approximately 10Hz, (Dawson and Fischer, 1994). The mean of a spectral estimator consists of the convolution of the true spectrum with a spectral window which depends on the particular estimation method used (e.g., Percival and Walden, 1993). The window will have a main lobe and multiple sidelobes and when the window is centred at the position of the spectral line, these sidelobes will transfer power into other parts of the spectrum including the delta band, even though the line is in the $[8,12] \mathrm{Hz}$ alpha band. If the line is sufficiently dominant, such side-lobe leakage will be noticeable and cause serious estimation errors in the delta band. As a result of such considerations, we 


\begin{tabular}{|c|c|c|c|c|c|c|c|c|c|}
\hline 1 & 2 & 3 & 4 & 5 & 6 & 7 & 8 & 9 & 10 \\
\hline F3 & F4 & C3 & C4 & P3 & P4 & O1 & O2 & T3 & T4 \\
\hline
\end{tabular}

Table 1: Series numbers corresponding to electrode positions.

applied a $6 \mathrm{~Hz}$ Butterworth low-pass filter to the EEG data before any spectral estimation of the delta band. Such a filter dramatically attenuates the $10 \mathrm{~Hz}$ line and and attendant effects. We also tried spectral line removal and spectral reshaping (e.g., Medkour et al., 2009), but found that the line varied slightly between individuals around $10 \mathrm{~Hz}$, and it was impossible to find a frequency grid on which the line fell for all individuals. The low-pass filtering produced better results and was consequently adopted.

The time series from the electrode sites were re-labelled as component processes $1-10$, respectively, i.e., $\left\{X_{j, t}\right\}, j=1, \ldots, 10$, as in Table 1 .

Nature of the data

The data was partitioned into $N_{b}=13$ epochs, each with sample size $N=$ 256. A typical ten-channel data set - incorporating the Butterworth filtering - is given in Fig. 1, (here for a negative-syndrome patient). For each epoch we assume that the data are segments from 10 jointly stationary processes. It might be reasonable to consider the epochs to be stationary segments from longer stationary time series, but this is not a necessary assumption for our approach. Our weaker assumption in this work is that the correlation structure of the 10 segments is invariant across epochs.

\section{Spectral estimation}

In this section we discuss statistical steps used in deriving partial coherence results for a particular individual and a particular epoch. As already mentioned we are concentrating on the low-frequency delta band covering the frequency range $[0.5,4] \mathrm{Hz}$. 


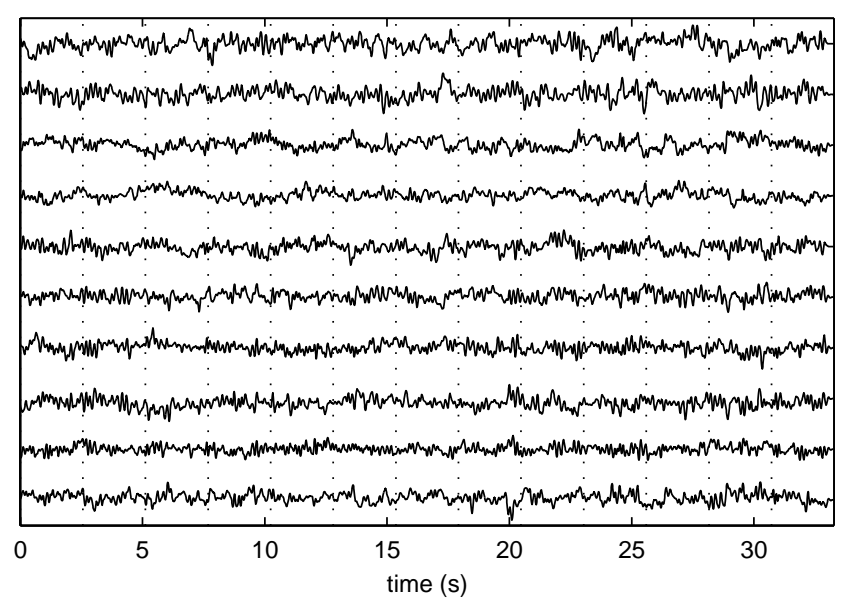

Figure 1: Ten-channel recorded data for a negative-syndrome patient after low-pass filtering to $6 \mathrm{~Hz}$. The epochs are delineated by the vertical dashed lines.

\section{The spectral matrix}

For a particular individual and a particular epoch we can assemble the EEG data into vector form as $\boldsymbol{X}_{t}=\left[X_{1, t}, \ldots, X_{p, t}\right]^{T}, t=0, \ldots, N-1$, where $p=10$ and $N=256$. We seek to estimate the spectral matrix

$$
\boldsymbol{S}(f)=\left[\begin{array}{cccc}
S_{11}(f) & S_{12}(f) & \ldots & S_{1 p}(f) \\
S_{21}(f) & S_{22}(f) & \ldots & S_{2 p}(f) \\
\vdots & \vdots & \ddots & \vdots \\
S_{p 1}(f) & S_{p 2}(f) & \ldots & S_{p p}(f)
\end{array}\right]
$$

for frequencies $f_{a} \leq f \leq f_{b}$, where $\left[f_{a}, f_{b}\right]=[0.5,4] \mathrm{Hz}$ for the delta band. The $l, m$ th term $S_{l m}(f)=(\boldsymbol{S}(f))_{l m}$ is the cross-spectrum for the $l$ and $m$ th processes. We carry out spectral estimation via the multitaper method (e.g., Percival and Walden, 1993). We make use of a set of $K$ real-valued orthonormal taper sequences $\left\{h_{k, t}, t=0, \ldots, N-1\right\}$, for $k=0, \ldots, K-1$. Since they are orthonormal we know $\sum_{t} h_{j, t} h_{k, t}=1$ if $j=k$, and zero otherwise.

We form the product $h_{k, t} \boldsymbol{X}_{t}$ of the $t$ th component of the $k$ th taper with the $t$ th component of the vector-valued process, and compute the (scaled) vector 
Fourier transform

$$
\boldsymbol{J}_{k}(f) \equiv \Delta t^{1 / 2} \sum_{t=0}^{N-1} h_{k, t} \boldsymbol{X}_{t} \mathrm{e}^{-\mathrm{i} 2 \pi f t \Delta t} .
$$

The estimator of the $p \times p$ spectral matrix $\boldsymbol{S}(f)$ is given by

$$
\hat{\boldsymbol{S}}(f)=\frac{1}{K} \sum_{k=0}^{K-1} \boldsymbol{J}_{k}(f) \boldsymbol{J}_{k}^{H}(f),
$$

where ${ }^{H}$ denotes transpose and complex conjugation.

An important choice is that of the number of tapers $K$ to use. Spectral matrices computed using $K$ sine tapers have an effective estimation bandwidth of $B=(K+1) /[(N+1) \Delta t] \mathrm{Hz}$, see e.g., Walden et al. (1995). The choice of $K=12$ here gives a bandwidth of $5 \mathrm{~Hz}$. It would be nice to reduce this, but any spectral estimation method we use requires that the number of complex degrees of freedom, $K$, satisfies $K \geq p$ in order that the spectral matrix is not singular, and therefore that distributions used in statistical hypothesis-testing are valid. Since $p=10$, the choice $K=12$ seems a reasonable compromise and this number of tapers was used in our analyses.

\section{Partial coherence}

The partial coherence between series $\left\{X_{j, t}\right\}$ and $\left\{X_{k, t}\right\}$, (with other processes held fixed), can be estimated as (Dahlhaus, 1997, 2000; Salvador et al., 2005)

$$
\hat{r}_{j k}(f) \equiv \frac{\left|\hat{S}^{j k}(f)\right|^{2}}{\hat{S}^{j j}(f) \hat{S}^{k k}(f)}, \quad f_{a} \leq f \leq f_{b},
$$

where $\hat{S}^{j k}(f)$ is the $(j, k)$ th term of the inverse spectral matrix, $\hat{\boldsymbol{S}}^{-1}(f)$. The partial coherence satisfies $0 \leq \hat{r}_{j k}(f) \leq 1$. In order to achieve stable inversion of the estimated spectral matrices we used the diagonal up-weighting scheme discussed in detail in Medkour et al. (2009) with up-weighting parameter $\zeta=$ $10^{-5}$; the shrinkage approach of Fiecas et al. (2010) is designed for the same purpose. 
In order to debias the raw estimated partial coherence we note the following. The statistical properties of the partial coherence estimator are those of ordinary coherence provided the degrees of freedom $K$ in ordinary coherence are replaced by $K-p+2$ for partial coherence. Making this adjustment in Benignus's formula (Benignus, 1969) for debiassing of ordinary coherence, we see that partial coherence can be debiassed by using

$$
\bar{r}_{j k}(f)=\frac{\hat{r}_{j k}(f)-\frac{1}{K-p+2}}{1-\frac{1}{K-p+2}} .
$$

For $K=12$ and $p=10$ a debiased version of the estimated partial coherence is thus given by

$$
\bar{r}_{j k}(f)=\frac{4}{3} \hat{r}_{j k}(f)-\frac{1}{3} .
$$

The formula (2) is used throughout this work when referring to the estimated partial coherence, apart from in hypothesis tests where of course the raw estimator is used since it is the distribution of the raw estimator which is known and utilised in the tests.

\section{Partial coherence and conditional correlation graphs}

A graph $G=(V, E)$ consists of vertices $V$ and edges $E$, where $E \subset\{(j, k) \in$ $V \times V: j \neq k\}$, i.e., edges connect pairs of distinct vertices. Our graphs are simple, there are neither loops from a vertex to itself nor any multiple edges between two vertices. Edges $(j, k) \in E$ for which both $(j, k) \in E$ and $(k, j) \in E$ are called undirected edges. If all edges are undirected, the graph is said to be an undirected graph, which is assumed here.

An edge is missing from the graph, i.e., $(j, k) \notin E$, if, and only if, $\left\{X_{j, t}\right\}$ and $\left\{X_{k, t}\right\}$ are uncorrelated given the other $(p-2)$ component processes. Note that there are $N_{i}=p(p-1) / 2$ possible connections between the series, or equivalently, edges to the graph. This is simply the number of off-diagonal terms in the upper (or lower) triangle of the spectral matrix.

$G=(V, E)$ defines an undirected conditional correlation graph and, for 


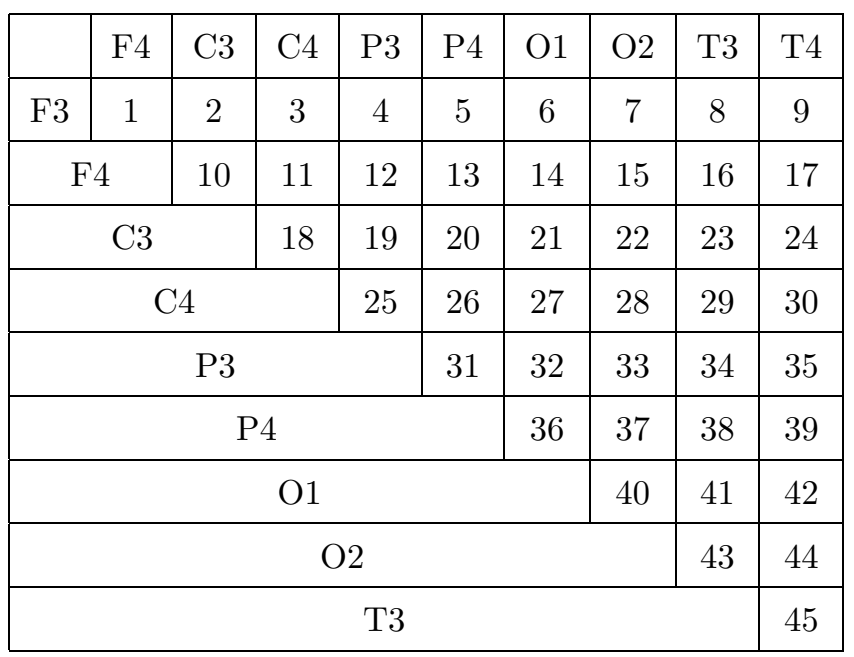

Table 2: Numbering (indices) of the connections.

Gaussian time series, a conditional independence graph. But (Dahlhaus, 2000)

$$
(j, k) \notin E \Leftrightarrow r_{j k}(f)=0, \quad f_{a} \leq|f| \leq f_{b},
$$

where $\Leftrightarrow$ denotes 'if and only if' and $r_{j k}(f)$ is the true value of the partial coherence at $f$. So to determine the graph, namely the presence or absence of edges, we need to test (3), making use of the estimate in (1).

In what follows, rather than using two indices $j, k$ to denote an edge we use the single index $i$ to enumerate a particular connection, as set out in Table 2 . Since $p=10$, the number of connection indices is $N_{i}=45$. So for example the presence or absence of an edge between F3 and C3 is equivalent to the presence or absence of connection $i=2$. The partial coherence may then be more simply written as $r_{i}(f)$.

Consider a particular connection $i$. It is impractical to try to conduct the test (3) at all frequencies in the continuum $f_{a} \leq f \leq f_{b}$. We therefore consider testing the hypotheses

$$
H_{l}: r_{i}\left(f_{l}\right)=0, \text { for all } l=1, \ldots, L,
$$

where $f_{a} \leq f_{1}<\cdots<f_{L} \leq f_{b}$ are a set of frequencies in the range $\left[f_{a}, f_{b}\right]$. 
(By symmetry we need only consider positive frequencies.) This is a multiple hypothesis testing problem. The test uses the known distribution of the raw partial coherence $\hat{r}_{i}(f)$ under the null hypothesis.

To carry out the multiple hypothesis testing we employ the stepdown method combined with the Holm method for determining the set of critical values employed. The Holm procedure is suitable for multiple hypothesis testing when the statistics being tested are correlated; this is the case here since the estimation bandwidth is $5 \mathrm{~Hz}$, so the estimators $\left\{\hat{r}_{i}\left(f_{l}\right), l=1, \ldots, L\right\}$, within the delta band, are obviously correlated. Full details of the Holm procedure may be found in Medkour et al. (2009); we required that the probability of one or more false rejections should not exceed 0.05 .

\section{Graphical model selection}

\section{Measuring strengths of connections}

We can determine the set of frequencies $\left\{f_{l^{\prime}}\right\}$, a subset of $\left\{f_{l}\right\}$, consisting of $L^{\prime}$ elements (possibly zero), at which the null hypothesis $H_{l}: r_{i}\left(f_{l}\right)=0$ is rejected. While only one hypothesis need be rejected to indicate an edge between $\left\{X_{j, t}\right\}$ and $\left\{X_{k, t}\right\}$, or connection $i$, the knowledge of the set of frequencies where rejection occurred gives us useful information of which we make use as follows. In Medkour et al. (2009) we used $L^{\prime} / L$, the ratio of the number of rejected hypotheses to the total tested, to measure the strength of connection $i$. Here we propose a weighted ratio measure where a rejected hypothesis at $f_{l^{\prime}}$ is weighted by the corresponding debiassed estimated partial coherence $\bar{r}_{i}\left(f_{l^{\prime}}\right)$ defined by (2). There are three data-set groupings, $\mathcal{P}, \mathcal{N}, \mathcal{C}$. For any grouping consider combining results over individuals, indexed by $a, a=1, \ldots, N_{a}$, and epochs, indexed by $b, b=1, \ldots, N_{b}$. We therefore define the weighted ratio measure by

$$
W R_{i}^{a b}=\frac{1}{L} \sum_{l^{\prime}=1}^{L^{\prime}} \bar{r}_{i}^{a b}\left(f_{l^{\prime}}\right) .
$$



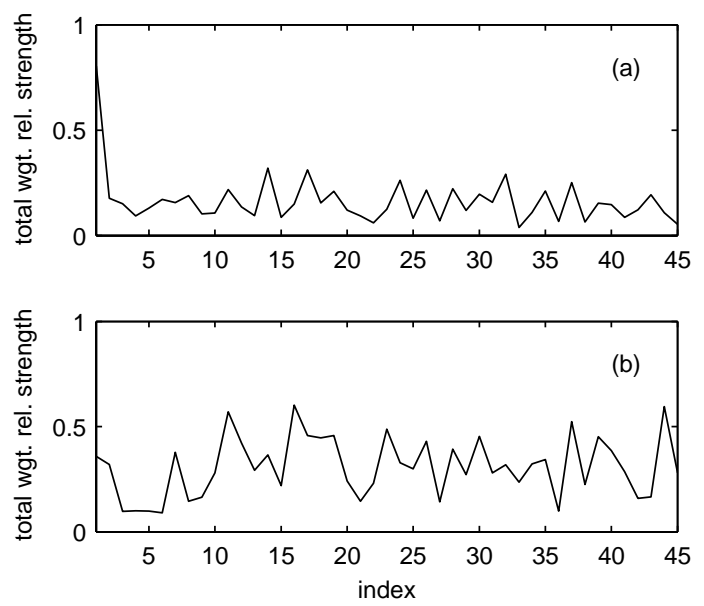

Figure 2: Total weighted relative strength, $W R S_{i}$, against connection index $i$, for (a) positivesyndrome patients, $\mathcal{P}$, and (b) negative-syndrome patients, $\mathcal{N}$.

Next we average $W R_{i}^{a b}$ over individuals (in the grouping) to obtain $\overline{W R}_{i}^{b}$. (This is our measure of the strength of the connection.) Now let $P I_{i}^{b}$ denote the proportion of individuals in the grouping which exhibit the connection $i$, i.e., (4) has been rejected. (This takes into account the incidence of the connection.) Then we define the weighted relative strength by

$$
W R S_{i}^{b}=\overline{W R}_{i}^{b} \cdot P I_{i}^{b}
$$

and finally take the sum over the $N_{b}$ epochs to obtain

$$
W R S_{i}=\sum_{b=1}^{N_{b}} W R S_{i}^{b}
$$

which is the total weighted relative strength for connection $i$. The quantity $W R S_{i}, i=1, \ldots, N_{i}$, with $N_{i}=45$, can be displayed as a vector. This is shown for the groupings $\mathcal{P}$ and $\mathcal{N}$ in Fig. 2.

For total weighted relative strength we now show how we chose a threshold $T$ to enable us to discern the most significant connections. 

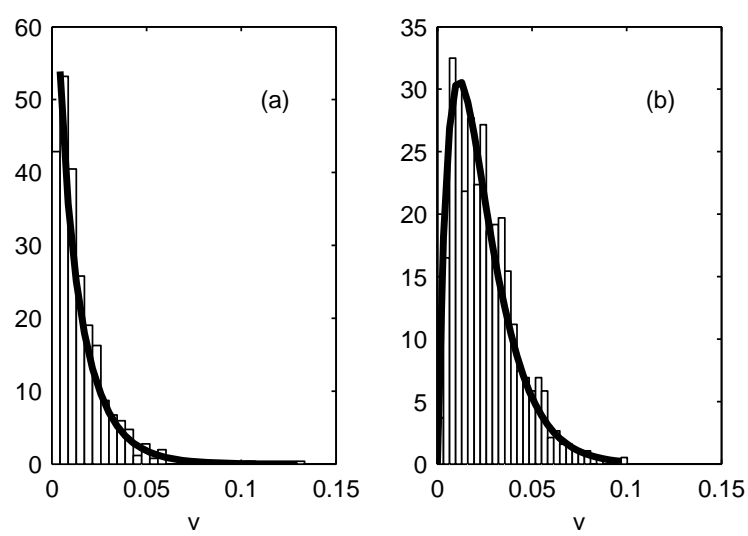

Figure 3: Empirical histogram for weighted relative strength plotted against fitted gamma PDF for (a) positive-syndrome patients and (b) negative-syndrome patients.

\section{A gamma distribution model for weighted relative strength}

For each of the groupings we now make the assumption that the values of $W R S_{i}^{b}$ over epochs and connections are realizations of independent and identically distributed (IID) random variables $V_{i}^{b}, i=1, \ldots N_{i}, b=1, \ldots, N_{b}$, each having the same distribution as

$$
V \sim \operatorname{gam}(\alpha, \beta)
$$

i.e., $V$ is gamma-distributed with probability density function (PDF) (Johnson and Kotz, 1994),

$$
f_{V}(v)=\frac{v^{\alpha-1}}{\Gamma(\alpha) \beta^{\alpha}} \exp [-(v / \beta)], \quad 0 \leq v \leq \infty,
$$

where $\alpha$ and $\beta$ are shape and scale parameters, and $\Gamma(\cdot)$ is the gamma function. The associated distribution function $(\mathrm{DF})$ is denoted $F_{V}(v)$. Parameter estimates for $\alpha$ and $\beta$ were obtained by maximum likelihood using the $N_{i} N_{b}=45 \times 13=585$ available samples. The maximum likelihood fit of the gamma distribution model to the data was tested by (a) comparing empirical/sample histograms to fitted/model PDFs, (b) comparing empirical DFs to fitted DFs, (c) comparing empirical probabilities to fitted probabilities, and (d) chi-square goodness-of-fit tests. 

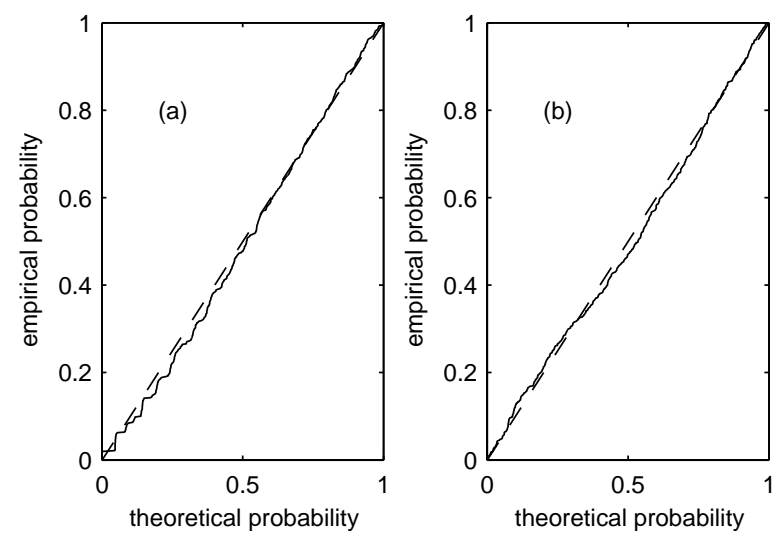

Figure 4: Gamma probability plots for (a) positive-syndrome patients and (b) negativesyndrome patients. The dashed line indicates ideal straight line behaviour.

The results shown here are for positive- and negative-syndrome patients, but all groupings display a good gamma distribution fit, with different parameter values for different groupings. A comparison of empirical histograms and fitted gamma PDFs is shown in Fig. 3. A probability plot for the gamma model is shown in Fig. 4, for which straight line behaviour is ideal. The excellent fits are clear to see. In both cases, chi-square goodness-of-fit tests did not reject the null hypothesis of a gamma distribution at the $5 \%$ level.

\section{A statistical model for total weighted relative strength}

We consider any one of the three data-set groupings. Total weighted relative strength $W R S_{i}$ was defined in (6). Under the gamma model, the values of $W R S_{i}^{b}$ over epochs and connections for the grouping are realizations of corresponding IID random variables $V_{i}^{b}, i=1, \ldots N_{i}, b=1, \ldots, N_{b}$, each having the same distribution as the gamma random variable $V(\alpha, \beta)$. This means that the $W R S_{i}, i=1, \ldots, N_{i}$, are realizations of corresponding IID random variables

$$
V_{i}=\sum_{b=1}^{N_{b}} V_{i}^{b}(\alpha, \beta),
$$

each having the same distribution as the random variable $V_{\Sigma}$, say, the sum of $N_{b}$ IID random variables each having the $\operatorname{gam}(\alpha, \beta)$ distribution. The result is 
well-known,

$$
V_{\Sigma} \sim \operatorname{gam}\left(N_{b} \alpha, \beta\right) .
$$

So the DF for $V_{\Sigma}$ is given by

$$
\begin{aligned}
F_{V_{\Sigma}}(v) & =\int_{0}^{v} \frac{y^{N_{b} \alpha-1}}{\Gamma\left(N_{b} \alpha\right) \beta^{N_{b} \alpha}} \exp [-(y / \beta)] \mathrm{d} y \\
& =\int_{0}^{v / \beta} \frac{u^{N_{b} \alpha-1}}{\Gamma\left(N_{b} \alpha\right)} \exp [-u] \mathrm{d} u \\
& =\Gamma\left(v / \beta, N_{b} \alpha\right)
\end{aligned}
$$

where $\Gamma(x, a)=[1 / \Gamma(a)] \int_{0}^{x} \mathrm{e}^{-t} t^{a-1} \mathrm{~d} t$ is the incomplete gamma function.

\section{Determining the graph}

The connectivity graph for any of the data-set groupings is determined by firstly specifying a threshold $T_{\rho}$ for total weighted relative strength as the quantile of the distribution of $V_{\Sigma}$ corresponding to a chosen probability $\rho$ found via the DF, i.e.,

$$
T_{\rho}=F_{V_{\Sigma}}^{-1}(\rho) .
$$

A connection vector $\boldsymbol{A}$ is produced, of length $N_{i}$ where the $i$ th entry is 1 if $W R S_{i}>T_{\rho}$ and zero otherwise. An edge is missing or present in the graph, i.e., $(j, k) \notin E$ or $(j, k) \in E$, respectively, if $A_{i}=0$ or $A_{i}=1$, respectively, where $i$ is the connection corresponding to $(j, k)$. Fig. 5 shows the result for the choice $\rho=0.9$. (The physical positions of the electrodes T3 and T4 have been deliberately displaced slightly downwards so that horizontal connections can be more readily distinguished.)

Note that a great strength of our gamma distribution model is that the only parameters involved in determining $F_{V_{\Sigma}}(v)$, (and hence $T_{\rho}$ ), namely $\alpha$ and $\beta$, are estimated from 585 samples, whereas in the absence of a distributional model the threshold for each $W R S_{i}, i=1, \ldots, N_{i}$, would need to be separately estimated from only $N_{b}=13$ sample values, a very undesirable situation. 


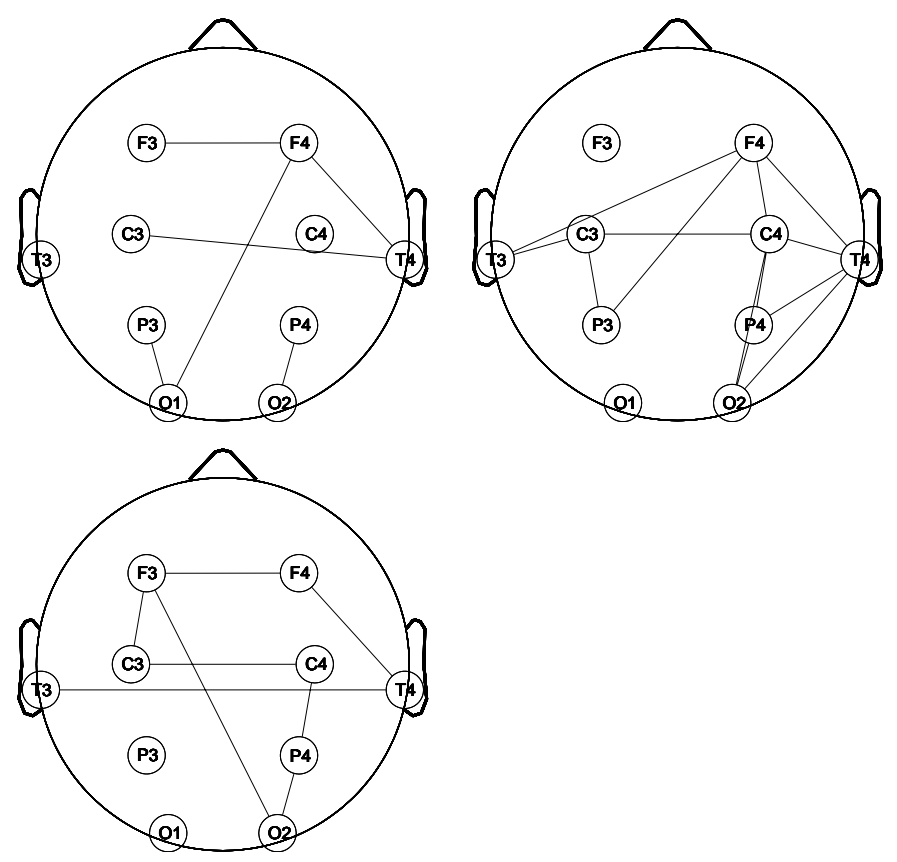

Figure 5: Interactions for positive-syndrome patients, (top left), negative-syndrome patients, (top right) and controls (bottom left), all for $\rho=0.9$. 


\section{Summary of steps}

For each grouping, $\mathcal{P}, \mathcal{N}, \mathcal{C}$, the following steps are carried out.

- Step 1: The spectral matrix $\hat{\boldsymbol{S}}^{a b}(f)$, say, is estimated for individuals $a=$ $1, \ldots, N_{a}$ and epochs $b=1, \ldots, N_{b}$.

- Step 2: The raw partial coherence, $\hat{r}_{i}^{a b}(f)$, for all possible connections $i=1, \ldots, N_{i}$ set out in Table II is estimated for individuals $a=1, \ldots, N_{a}$ and epochs $b=1, \ldots, N_{b}$. From this a debiassed version, $\bar{r}_{i}^{a b}(f)$, is found from (2). For each connection $i$ the Holm stepdown procedure determines the number $L^{\prime}$ (possibly null) and set of frequencies $\left\{f_{l^{\prime}}\right\}$ (possibly empty) for which the hypothesis of null partial coherence is rejected.

- Step 3: The weighted ratios $W R_{i}^{a b}$, defined in (5), are then computed and averaged over individuals $a=1, \ldots, N_{a}$ in the grouping to give $\overline{W R}_{i}^{b}$.

- Step 4: The proportion of individuals in the grouping which exhibit connection $i$, namely $P I_{i}^{b}$ is found next. Then the weighted relative strength is found from $W R S_{i}^{b}=\overline{W R}_{i}^{b} \cdot P I_{i}^{b}$.

- Step 5: The $W R S_{i}^{b}$ values are well-fitted by a gamma distribution with parameters $\alpha$ and $\beta$ estimated by maximum likelihood, whence $W R S_{i}=$ $\sum_{b=1}^{N_{b}} W R S_{i}^{b}, i=1, \ldots, N_{i}$, is a sample from a gamma distribution with parameters $N_{b} \alpha$ and $\beta . W R S_{i}$ is the total weighted relative strength for connection $i$.

- Step 6: For a chosen probability $\rho$ a threshold $T_{\rho}$ for total weighted relative strength is determined as the corresponding quantile of the $\operatorname{gam}\left(N_{b} \alpha, \beta\right)$ distribution. For the grouping under consideration, a connection vector $\boldsymbol{A}$ is produced, of length $N_{i}$ where the $i$ th entry is 1 if $W R S_{i}>T_{\rho}$ and zero otherwise.

- Step 7: The graph is constructed using the rule that an edge is present in the graph, i.e., $(j, k) \in E$, if $A_{i}=1$. The graph depends on the choice of $\rho$. 


\begin{tabular}{|c|c|c|c|c|c|}
\hline$\rho$ & 0.5 & 0.8 & 0.9 & 0.95 & 0.99 \\
\hline \hline $\mathcal{P} \& \mathcal{C}$ & 29 & 24 & 18 & 16 & 9 \\
\hline $\mathcal{N} \& \mathcal{C}$ & 38 & 31 & 29 & 27 & 22 \\
\hline
\end{tabular}

Table 3: Hamming distance (\%) of graphs of positive- and negative-syndrome patients from the graph for controls. The threshold percentile $\rho$ is defined in (7).

\section{Distances between graphs}

\section{Hamming distances}

For each of our groupings, and a chosen $\rho$, we have a connection vector $\boldsymbol{A}$, of length $N_{i}=45$, where we assign unity where a connection has been detected by exceedance of threshold $T_{\rho}$ and zero otherwise. We can compare two graphs by comparing their corresponding connection vectors. The Hamming distance measures the percentage of vector entries that differ and has been used elsewhere for measuring the distance between graphs (e.g., Talih and Hengartner, 2005).

Suppose we have three connection vectors $\boldsymbol{A}, \boldsymbol{B}$ and $\boldsymbol{C}$. and let $\operatorname{Ham}\{\boldsymbol{A}, \boldsymbol{B}\}$ denote the Hamming distance between $\boldsymbol{A}$ and $\boldsymbol{B}$, i.e., the percentage of vector entries which differ. It is important in what follows to note that $\operatorname{Ham}\{\boldsymbol{A}, \boldsymbol{B}\}=$ $\operatorname{Ham}\{\boldsymbol{A}, \boldsymbol{C}\} \nRightarrow \boldsymbol{B}=\boldsymbol{C}$, it merely tells us that $\boldsymbol{B}$ and $\boldsymbol{C}$ differ from $\boldsymbol{A}$ by the same percentage of entries, but not necessarily the same ones. Contrariwise, $\operatorname{Ham}\{\boldsymbol{A}, \boldsymbol{B}\} \neq \operatorname{Ham}\{\boldsymbol{A}, \boldsymbol{C}\} \Rightarrow \boldsymbol{B} \neq \boldsymbol{C}$. In other words, the Hamming distance is suited for detecting dissimilarity.

Using this distance measure we compared firstly the graph of positive-syndrome patients to the graph of controls, and secondly the graph of negative-syndrome patients to the graph of controls. This was done for threshold levels corresponding to $\rho=0.5,0.8,0.9,0.95$ and 0.99 . The results are shown in Table 3 . The graph for negative-syndrome patients is further from the graph for controls than is the graph for positive-syndrome patients, for all the values of $\rho$ considered.

This is a very interesting result, but we need to show that such differences are statistically significant. We shall use the statistical technique of bootstrapping 
to investigate this.

\section{Bootstrapping and $t$ test}

Given our assumption that the multi-channel correlation structure is invariant across epochs, the epochs can be treated as exchangeable in our application in the same sense that elements of a random sample are treated as such in the standard bootstrapping scheme (Efron and Tibshirani, 1993).

For each grouping, and given individual $a$ and connection $i$, let us denote the indices of the epochs used in our scheme up to now by $b=b_{1}, \ldots, b_{N_{b}}$, where $b_{j}=j$. With bootstrapping we draw a random sample of size $N_{b}$ from $b_{1}, \ldots, b_{N_{b}}$, with replacement. We denote these indices by $b^{*}=b_{1}^{*}, \ldots, b_{N_{b}}^{*}$, a list which may contain some integers in the range $1, \ldots, N_{b}$ more than once, and others not at all. Analysis steps 3 to 7 are modified as follows (unchanged details are excluded):

- Step $3^{*}$ : We draw the sample $b^{*}=b_{1}^{*}, \ldots, b_{N_{b}}^{*}$. The weighted ratios $W R_{i}^{a b^{*}}, b^{*}=b_{1}^{*}, \ldots, b_{N_{b}}^{*}$, are averaged over individuals $a=1, \ldots, N_{a}$ in the grouping to give $\overline{W R}_{i}^{b^{*}}$.

- Step $4^{*}$ : The proportion of individuals in the grouping which exhibit connection $i$, namely $P I_{i}^{b^{*}}$ is found next. Then the weighted relative strength is found from $W R S_{i}^{b^{*}}=\overline{W R}_{i}^{b^{*}} \cdot P I_{i}^{b^{*}}$, for $b^{*}=b_{1}^{*}, \ldots, b_{N_{b}}^{*}, i=1, \ldots, N_{i}$.

- Step $5^{*}: W R S_{i}=\sum_{b^{*}} W R S_{i}^{b^{*}}, i=1, \ldots, N_{i}$, is a sample from a gamma distribution with parameters $N_{b} \alpha$ and $\beta$.

- Step $6^{*}$ : For the grouping under consideration, a connection vector $\boldsymbol{A}_{1}^{*}$ is produced, of length $N_{i}$ where the $i$ th entry is 1 if $W R S_{i}>T_{\rho}$ and zero otherwise.

- Step $7^{*}$ : The graph is constructed using the rule that connection $i$ is present in the graph, i.e., $(j, k) \in E$, if $A_{1, i}^{*}=1$.

Steps $3^{*}$ to $7^{*}$ are repeated a large number $N_{r}=5000$ times to obtain connection vectors $\boldsymbol{A}_{1}^{\mathcal{P} *}, \ldots, \boldsymbol{A}_{N_{r}}^{\mathcal{P} *}$ for patients diagnosed with positive syndrome, and then 

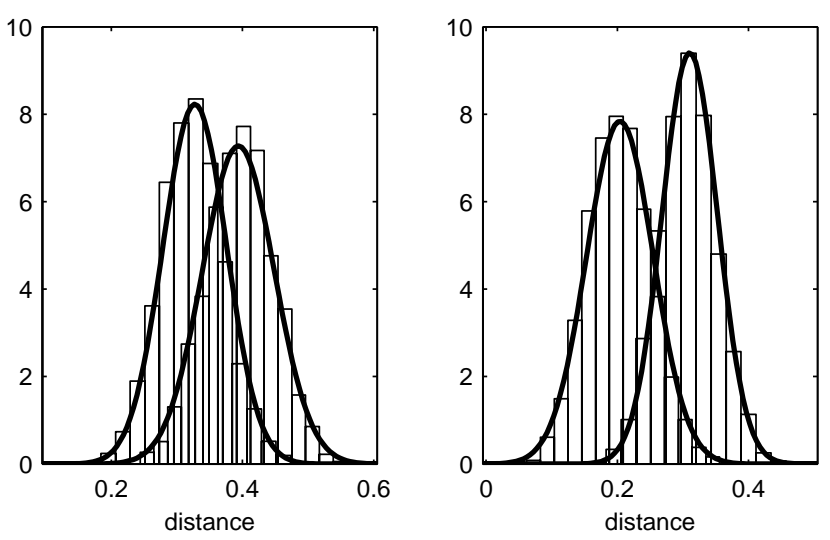

Figure 6: Bootstrap histograms of the Hamming distances $D_{j}^{\mathcal{P}, \mathcal{C}}$ (left-most histogram and distribution) and $D_{j}^{\mathcal{N}, \mathcal{C}}$ (right-most histogram and distribution) for $\rho=0.5$ (left plot) and 0.9 (right plot).

repeated, independently, $N_{r}$ times to obtain connection vectors $\boldsymbol{A}_{1}^{\mathcal{C} *}, \ldots, \boldsymbol{A}_{N_{r}}^{\mathcal{C} *}$ for controls. For each boostrap sample, $j=1, \ldots, N_{r}$, we calculate the Hamming distances

$$
D_{j}^{\mathcal{P}, \mathcal{C}}=\operatorname{Ham}\left\{\boldsymbol{A}_{j}^{\mathcal{P} *}, \boldsymbol{A}_{j}^{\mathcal{C} *}\right\} .
$$

This approach could be considered a two-sample bootstrapping problem akin to that discussed in Efron and Tibshirani (1993, p. 88).

The whole bootstrapping scheme is repeated, independently, to obtain connection vectors $\boldsymbol{A}_{1}^{\mathcal{N} *}, \ldots, \boldsymbol{A}_{N_{r}}^{\mathcal{N} *}$ for patients diagnosed with negative syndrome, and $\boldsymbol{A}_{1}^{\mathcal{C} *}, \ldots, \boldsymbol{A}_{N_{r}}^{\mathcal{C} *}$ for controls. For each boostrap sample, $j=1, \ldots, N_{r}$, we calculate the Hamming distances

$$
D_{j}^{\mathcal{N}, \mathcal{C}}=\operatorname{Ham}\left\{\boldsymbol{A}_{j}^{\mathcal{N} *}, \boldsymbol{A}_{j}^{\mathcal{C} *}\right\}
$$

From the 5000 samples of $D_{j}^{\mathcal{P}, \mathcal{C}}$ and 5000 independent samples of $D_{j}^{\mathcal{N}, \mathcal{C}}$ thus calculated, histograms are readily produced.

This whole process was done for each of the percentage points in Table 3.

By way of example, these distances are shown for $\rho=0.5$ in the left plot of Fig. 6 and for $\rho=0.9$ in the right plot. In each plot the left-most histogram 


\begin{tabular}{|c|c|c|c|c|c|}
\hline$\rho$ & 0.5 & 0.8 & 0.9 & 0.95 & 0.99 \\
\hline \hline Statistic value & 64 & 95 & 113 & 132 & 170 \\
\hline Degrees of freedom & 9850 & 9887 & 9685 & 9988 & 9953 \\
\hline
\end{tabular}

Table 4: Two-sample $t$ test statistic values and corresponding degrees of freedom for different threshold percentiles $\rho$ defined in (7).

with associated fitted normal distribution is for the Hamming distances $D_{j}^{\mathcal{P}, \mathcal{C}}$ and the right-most histogram with associated fitted normal distribution is for $D_{j}^{\mathcal{N}, \mathcal{C}}$. The normal distribution fits are excellent, and enable us to carry out a two-sample $t$ test of the null hypothesis $H_{0}: \mu^{\mathcal{P}, \mathcal{C}}=\mu^{\mathcal{N}, \mathcal{C}}$, where $\mu^{\mathcal{P}, \mathcal{C}}$ is the mean distance between graphs of positive-syndrome patients and controls, and likewise for $\mu^{\mathcal{N}, \mathcal{C}}$. The problem of making inference on means when variances are not assumed equal is known as the Behren's-Fisher problem and the form of the test statistic is given in Casella and Berger (1990, pp. 396-7) where it is pointed out that the degrees of freedom may be calculated by Satterthwaite's method. The results are given in Table 4 . Since the $t$ distribution is essentially the same as the normal for degrees of freedom exceeding 1000, we can compare the values of the statistic to normal percentiles and we see that the results are extremely significant. The null hypothesis is clearly rejected in favour of $\mu^{\mathcal{P}, \mathcal{C}}<\mu^{\mathcal{N}, \mathcal{C}}$.

\section{Concluding discussion}

Although there have been previous reports of differences in the patterns of functional connectivity between patients with positive and negative schizophrenia using EEG, these have invariably been confounded either by differences in medication, measurement issues (e.g. volume conduction) or both. To overcome these problems we have used a sample of unmedicated patients and a data-driven approach using well-established statistical modelling and estimation steps based around the partial coherence approach of Medkour et al. (2009).

This approach to functional connectivity has many advantages over existing methods and could, in principle, be applied to any brain mapping method that 
generates time-series data. The greatest benefits, however, are likely to be seen in EEG or MEG studies. Currently, the method is limited to cases of graphs with relatively few nodes: the non-singularity of estimated spectral matrices is a key requirement, and the current authors are considering ways to deal with this problem for dense array EEG/MEG data in sensor space. Prior reduction of the dimensionality of the data by, for example, source localisation, would also be a valid approach.

With the presented methodological advances, we have been able to show for the first time that there are reliable differences in the patterns of functional connectivity between patients with positive and negative schizophrenia. Our results indicate that the brain connectivity graphs for patients diagnosed with negative syndrome are on average further from the graphs of controls than are the brain connectivity graphs for patients diagnosed with positive syndrome. This is consistent with the idea that the negative syndrome is a more severe form of schizophrenia with symptoms entailing greater disability and poorer prognosis (Fenton and McGlashan, 1991).

These findings are not, however, consistent with the concept of schizophrenia as a simple disconnection syndrome. For neither syndrome was it the case that the patients' graphs were simply diminished versions of the controls'. Rather, our findings support the more general notion of a 'dysconnection' syndrome (Stephan et al., 2006) whereby schizophrenia is characterised not only by the absence of functional connectivity but also by the presence of aberrant connections. Why might this be? Firstly, the measure of connectivity we use is functional, not structural. Patients might have a normal brain structure but be using their brain in a non-standard way. For example, in recovery from brain injury, some improvement results from patients solving a problem by using a different strategy (i.e., one that does not require the damaged part of the brain). Something comparable might be going on here, namely patients using different cognitive strategies to controls. Secondly, in normal development, the brain starts with far more neurones and synaptic connections than it needs and around the time of adolesence, there is massive synaptic pruning leading to a loss 
of many neurones. Pruning is based on the synaptic strength which is derived from how many times each synapse has been activated: more activation, more synaptic strength. In schizophrenia, abnormal development leads to atypical development of synaptic strengths which is latent in childhood because of the massive redundancy of neural tissue. However, in schizophrenia, because of the abnormal synaptic strengths, when the pruning process starts, some connections that are useful are lost, and others that are aberrant will be kept.

Given the well-known spatial limitations of EEG, the present results have little to say about the localisation of the brain regions that gave rise to the dysconnections observed. However connections were seen in the frontal, temporal, parietal and occipital regions and in both the left and right hemispheres. Furthermore, multiple long-range connections were found both between lobes and between hemispheres. The implication of this must be that schizophrenia, and its major subtypes, are characterised by widespread abnormalities in the pattern of connectivity.

\section{Acknowledgements}

The work of TM, ATW and APB was supported by the Engineering and Physical Science Research Council (UK) and the Medical Research Council (UK) and that of VBS by grants from the Russian Foundation for Humanities (No. 09-06-00-459a) and from the BIAL Foundation (No. 20/02). Helpful suggestions by the referees were much appreciated.

\section{References}

Andreasen, N. C., Olsen, S. A., 1982. Negative v positive schizophrenia: Definition and validation. Archives of General Psychiatry 39, 789-794.

Andreasen, N. C., 1983. The Scale for the Assessment of Negative Symptoms (SANS). The University of Iowa, Iowa City, IA. 
Andreasen, N. C., 1984. The Scale for the Assessment of Positive Symptoms (SAPS). The University of Iowa, Iowa City, IA.

Begić, D., Hotujac, L., Jokić-Begić, N., 2000. Quantitative EEG in 'positive' and 'negative' schizophrenia. Acta Psychiatrica Scandinavica, 101, 307-311.

Begić, D., Mahnik-Miloš, M., Grubišin, J., 2009. EEG characteristics in depression, 'negative' and 'positive' and schizophrenia. Psychiatria Danubina 21, $579-584$

Benignus, V. A., 1969. Estimation of the coherence spectrum and its confidence interval using the fast Fourier transform. IEEE Trans. Audio and Electroacoustics $17,145-150$.

Bullmore, E., Fletcher, P., 2003. The eye's mind: brain mapping and psychiatry. British J. Psychiatry 182, 381-384.

Casella, G., Berger, R. L., 1990. Statistical Inference. Wadsworth, Inc., Belmont CA.

Coutin-Churchman, P., Añez, Y., Uzcátegui, M., Alvarez, L., Vergara, F., Mendez, L. and Fleitas, R., 2003. Quantitative spectral analysis of EEG in psychiatry revisited: drawing signs out of numbers in a clinical setting. Clin. Neurophysiology 114, 2294-2306.

Dahlhaus, R., 2000. Graphical interaction models for multivariate time series. Metrika 51, 157-72.

Dahlhaus, R., Eichler, M., Sandkühler, J., 1997. Identification of synaptic connections in neural ensembles by graphical models. J. Neurosci. Meth. 77, 93107.

Dawson, G., Fischer, K.W., 1994. Human Behavior and the Developing Brain. The Guilford Press.

Efron, B., Tibshirani, R. J., 1993. An Introduction to the Bootstrap. Chapman \& Hall/CRC. 
Fenton, W. S., McGlashan, T.H., 1991. Natural history of schizophrenia subtypes II: positive and negative symptoms and long-term course. Arch. Gen. Psychiatry. 48, 978-986.

Fiecas, M., Ombao, H., Linkletter, C., Thompson, W., Sanes, J., 2010. Functional connectivity: shrinkage estimation and randomization test. NeuroImage 49, 3005-3014.

Friston K. J., Frith, C. D., 1995. Schizophrenia: a disconnection syndrome? Clin. Neurosci. 3, 89-97.

Galderisi, S., Mucci, A., Volpe, U., Boutros, N., 2009. Evidence-based medicine and electrophysiology in schizophrenia. Clin. EEG Neurosci. 40, 62-77.

Gross, A., Joutsiniemi, S-L., Rimon, R., Appelberg, B., 2006. Correlation of symptom clusters of schizophrenia with absolute powers of main frequency bands in quantitative EEG. Behavioral and Brain Functions 2, 23. doi:10.1186/1744-9081-2-23.

Harris, A. W. F., Bahramali, H., Slewa-Younan, S., Gordon, E., Williams, L., Li W. M., 2001. The topography of quantified electroencephalography in three syndromes of schizophrenia. Int. J. Neurosci. 107, 265-278.

John, E. R., Prichep, L. S., Alper, K. R., Mas, F. G., Cancro, R., Easton, P., Sverdlov, L., 1994. Quantitative electrophysiological characteristics and subtyping of schizophrenia. Biol. Psychiatry 36, 801-826.

John, J.P., Rangaswamy, M., Thennarasu, K., Khanna, S., Nagaraj, R. B., Mukundan, C.R., Pradhan, N., 2009. EEG power spectra differentiate positive and negative subgroups in neuroleptic-naive schizophrenia patients. J. Neuropsychiatry Clin. Neurosci. 21, 160-172.

Johnson, N. L., Kotz, S., Balakrishnan, N., 1994. Continuous Univariate Distributions, 2nd ed., vol. 1: Models and Applications. John Wiley \& Sons, Inc., New York. 
Lee, K-H., Williams, L. M., Breakspear, M., Gordon, E., 2003. Synchronous gamma activity: A review and contribution to an integrative neuroscience model of schizophrenia. Brain Res. Rev. 41, 57-78.

Leocani, L., Comi G., 1999. EEG coherence in pathological conditions. J. Clin. Neurophysiol. 16, 548-555.

Medkour, T., Walden, A. T., Burgess, A., 2009. Graphical modelling for brain connectivity via partial coherence. J. Neurosci. Meth. 180, 374-383.

Nolte, G., Ou, B., Wheaton, L., Mari, Z., Vorbach, S., Hallett, M., 2004. Identifying true brain interaction from EEG data using the imaginary part of coherency. Clin. Neurophysiol. 115, 2292-2307.

Percival, D. B., Walden, A. T., 1993. Spectral Analysis for Physical Applications. Cambridge University Press, Cambridge UK.

Salvador, R., Suckling, J., Schwarzbauer, C., Bullmore, E., 2005. Undirected graphs of frequency-dependent functional connectivity in whole brain networks. Phil. Trans. Roy. Soc. Lond., Ser. B, 360, 937-46.

Stam, C. J., Nolte, G., Daffertshofer, A., 2007. Phase lag index: assessment of functional connectivity from multichannel EEG and MEG with diminished bias from common sources. Hum. Brain Mapp. 28, 1178-1193.

Stephan, K. E., Baldeweg, T., Friston, K. J., 2006. Synaptic plasticity and dysconnection in schizophrenia. Biological Psychiatry 59, 929-939.

Strelets, V. B., Novototsky-Vlasov, V. Y., Golikova, J. V., 2002. Cortical connectivity in high frequency beta-rhythm in schizophrenics with positive and negative symptoms. Int. J. Psychophysiol. 44, 101-115.

Talih, M., Hengartner, N., 2005. Structural learning with time-varying components: tracking the cross-section of financial time series. J. Roy. Statist. Soc. Ser. B, 67, 321-341. 
Uhlhaas, P.J., Haenschel, C., Nikolić, D., Singer, W., 2008. The role of oscillations and synchrony in cortical networks and their putative relevance for the pathophysiology of schizophrenia. Schizophrenia Bull. 34, 927-943.

Walden, A. T., McCoy, E. J., Percival, D. B., 1995. The effective bandwidth of a multitaper spectral estimator. Biometrika 82, 201-14. 